Article

\title{
Radio-to- $\gamma$-Ray, Broadband Variability Study of the Classical BL Lac Object PKS 0735+178
}

\author{
Arti Goyal ${ }^{1, *}$, tukasz Stawarz ${ }^{1}$, Michal Ostrowski ${ }^{1}$ and Valeri Larionov ${ }^{2}$ \\ 1 Astronomical Observatory, Jagiellonian University, ul. Orla 171, Krakow 30-244, Poland; \\ stawarz@oa.uj.edu.pl (Ł.S.); mio@oa.uj.edu.pl (M.O.) \\ 2 Astronomical Institute of St. Petersburg State University, Petrodvorets 198504, Russia; vlar@astro.spbu.ru \\ * Correspondence: arti@oa.uj.edu.pl; Tel.: +48-12-6238-654
}

Academic Editors: Jose L. Gómez, Alan P. Marscher and Svetlana G. Jorstad

Received: 18 July 2016; Accepted: 23 September 2016; Published: 10 October 2016

\begin{abstract}
The power-law shape of the power spectral density (PSD) of blazar light curves- $P\left(v_{k}\right) \propto v_{k}^{-\beta}$, where $v_{k}$ is the temporal frequency-indicates that blazar variability is, in general, of the colored-type noise $(\beta \simeq 1-3)$. A precise characterisation of PSD slopes, normalizations, or characteristic timescales (if any) manifesting as distinct features in the power spectra of blazars is important for constraining the physics of the emission and energy dissipation processes in relativistic jets. Here we present the results of the PSD analysis for the BL Lac object PKS 0735+178 at GeV (Fermi-LAT), optical (R-band), and radio ( $\mathrm{GHz}$ band from UMRAO and OVRO programmes) frequencies, covering a broad range in variability timescales. The novelty of our approach is that in the optical regime, by combining the long-term and densely sampled R-band intra-night light curves, we constructed the PSD for time periods ranging from 23 years down to minutes. Our analysis reveals that: (1) the nature of processes generating flux variability at optical and radio frequencies is different from those operating at $\mathrm{GeV}$ photon energies ( $\beta \sim 2$ and 1, respectively); (2) the main driver behind the optical variability is the same on timescales of years, months, days, and hours (a single power-law with $\beta \sim 2$ ). We discuss our findings in the framework of a model where the overall blazar variability is generated by an underlying single stochastic process (radio and optical frequencies), or a linear superposition of such processes ( $\gamma$-ray regime).
\end{abstract}

Keywords: galaxies: active; BL Lacertae objects: individual (PKS 0735+178); variability

\section{Introduction}

The highly variable radio-to-optical/X-ray emission of blazar sources results from the synchrotron radiation of ultrarelativistic electrons in a highly-magnetized relativistic jet. The high-energy $\mathrm{X}$-ray $/ \gamma$-ray blazar emission continua, on the other hand, are most widely believed to be due to the inverse-Compton (IC) up-scattering of various photon fields by the same or distinct electron population [1]. Intensity changes of a few up to tens of percent occurring on timescales less than a day-commonly observed in blazars at radio and optical frequencies-are known as intra-night variability (INV), or a microvariability. The origin of such (particularly in relation to large-amplitude but typically longer-timescale variability observed most pronouncedly in $\gamma$-rays) is still being widely debated. Several competing scenarios have been proposed to explain the observed blazar variability, either a low- or high-amplitude one, some involving even purely geometrical effects (e.g., the "light house effect" where precession of jets causes differential forward beaming of the emission [2]). A set of widely accepted "intrinsic origin" models, favoured due to the results of the polarimetric studies revealing correlated changes in the fractional polarization (PD) and a concomitant swing in the polarization angle $(\chi)$ advocates instead for various plasma instabilities leading to the formation of shocks, magnetic reconnection sites, and turbulence in the jet flow, and hence to heating and 
accelerating of the jet particles [3-5]. Detailed analysis of blazar power spectral densities (PSDs) at various frequencies and variability timescales may, in principle, help us to discriminate between those various potential "intrinsic" causes.

The present study focuses on exploring the multi-wavelength (radio-to- $\gamma$-ray) broadband (decades-to-minutes) variability characteristics of the BL Lac object PKS 0735+178, singled out from the blazar class due to its persistent lack of intra-night optical variability; i.e., INV duty cycle DC $\sim 0$ for variability amplitudes $\psi>3 \%$ estimated using 17 nights of data spanning over 11 years of monitoring [6].

\section{Methodology: Generation of Light Curves and PSD Analysis}

The $\gamma$-ray light curve of PKS $0735+178$ was generated using the Fermi-LAT data for the photon energies 0.1-200 GeV, starting from August 2008 until September 2015, with the standard unbinned likelihood analysis (http://fermi.gsfc.nasa.gov/ssc/data/analysis/scitools/). The daily averaged R-band photo-polarimetric data were obtained using (1) $70 \mathrm{~cm}$ AZT-8 telescope of the Crimean Astrophysical Observatory (Nauchnij, Ukraine); (2) $40 \mathrm{~cm}$ LX-200 telescope of St. Petersburg State University, and $1.8 \mathrm{~m}$ Perkins telescope of Lowell Observatory (Flagstaff, AZ, USA); (3) $1.54 \mathrm{~m}$ Kuiper and $2.3 \mathrm{~m}$ Bok telescopes of Steward Observatory (Mt. Bigelow and Kitt Peak, AZ, USA); and (4) $2.2 \mathrm{~m}$ telescope of the Calar Alto Observatory within the MAPCAT program (http://www.iaa.es/ iagudo/research/MAPCAT/MAPCAT.html). The photometric data are also supplemented with the nightly averaged R-band optical data starting from 1993 until 2005, obtained from [7]. The R-band magnitude was calibrated using the standard stars from [7] with an accuracy of $\leq 0.2-0.5$ percent and converted to flux using zero point $3064 \mathrm{Jy}$. For details regarding optical R-band intra-night observations, see [8]. The radio data were obtained from the $26 \mathrm{~m}$ University of Michigan Radio Astronomy Observatory (UMRAO https:/ / dept.astro.lsa.umich.edu/datasets/umrao.php) dish at $4.8,8$, and $14.5 \mathrm{GHz}$ frequencies, and the $40 \mathrm{~m}$ Telescope at the Owens Valley Radio Observatory (OVRO (http://www.astro.caltech.edu/ovroblazars/)) at $15 \mathrm{GHz}$. For all of those lightcurves, the corresponding PSDs were generated following [9].

\section{Results}

Figure 1 presents the resulting PSDs of PKS $0735+178$ at radio ( 15 and $4.8 \mathrm{GHz}$; left and middle panels, respectively) and $\gamma$-ray $(0.1-200 \mathrm{GeV}$; right panel) photon energies. Figure 2 presents the optical (R-band) PSD of the source, including both the LTV and the INV datasets.

\section{Conclusions}

(1) The newly obtained long-term Fermi-LAT and optical data reveal intensity changes by a factor of a few on timescales of months, while the OVRO data show a steady increase in intensity by the same amount, though over a time span of 3 years. The long-term photo-polarimetric data show large variations in the optical EVPA (traced during 2005-2015) on timescales of weeks/months and years, which seem uncorrelated with the optical polarization degree and with the optical flux changes (Figure 3). All the gathered photo-polarimetric data do not support a "grand design" helical magnetic field structure in the PKS $0735+178$ jet (in contrast to [10]), as the $\chi_{o p t}$ and $P D_{o p t}$ changes observed on timescales of weeks-to-years are erratic and uncorrelated with flux changes. The jet therefore seems to be fully turbulent, with un-coupled perturbations in the bulk velocity, particle density, and magnetic field driving all together the uncorrelated flux-EVPA-PD variability. 


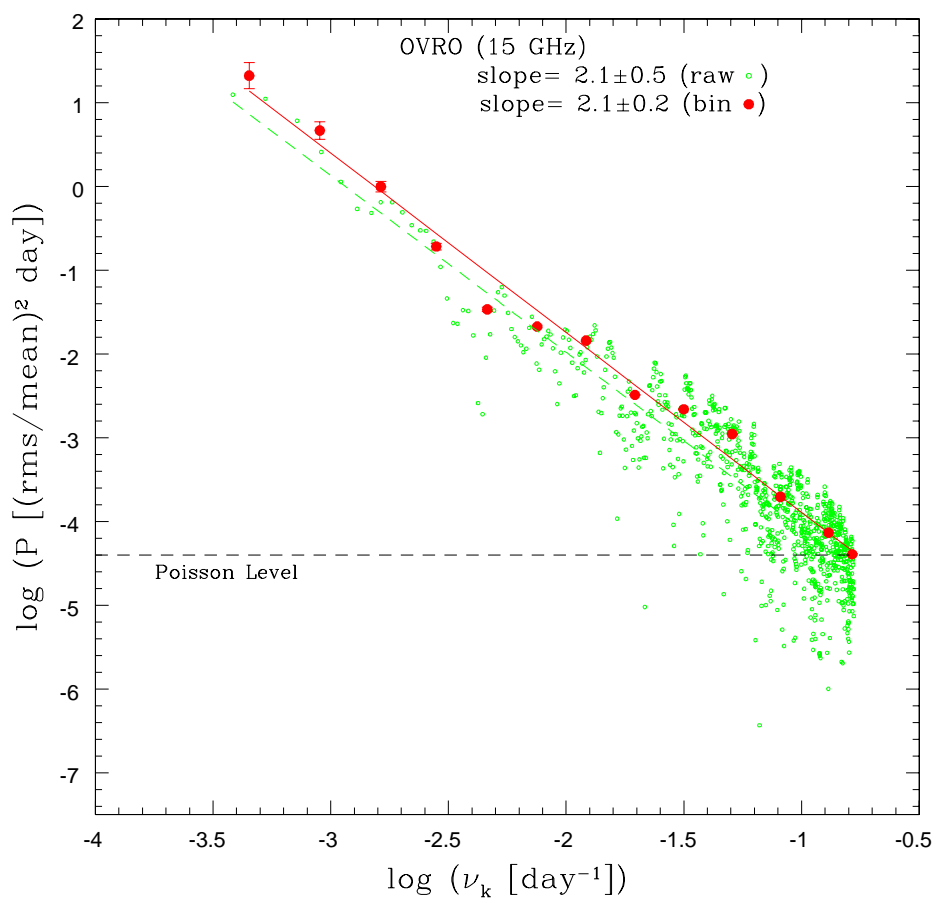

(a)

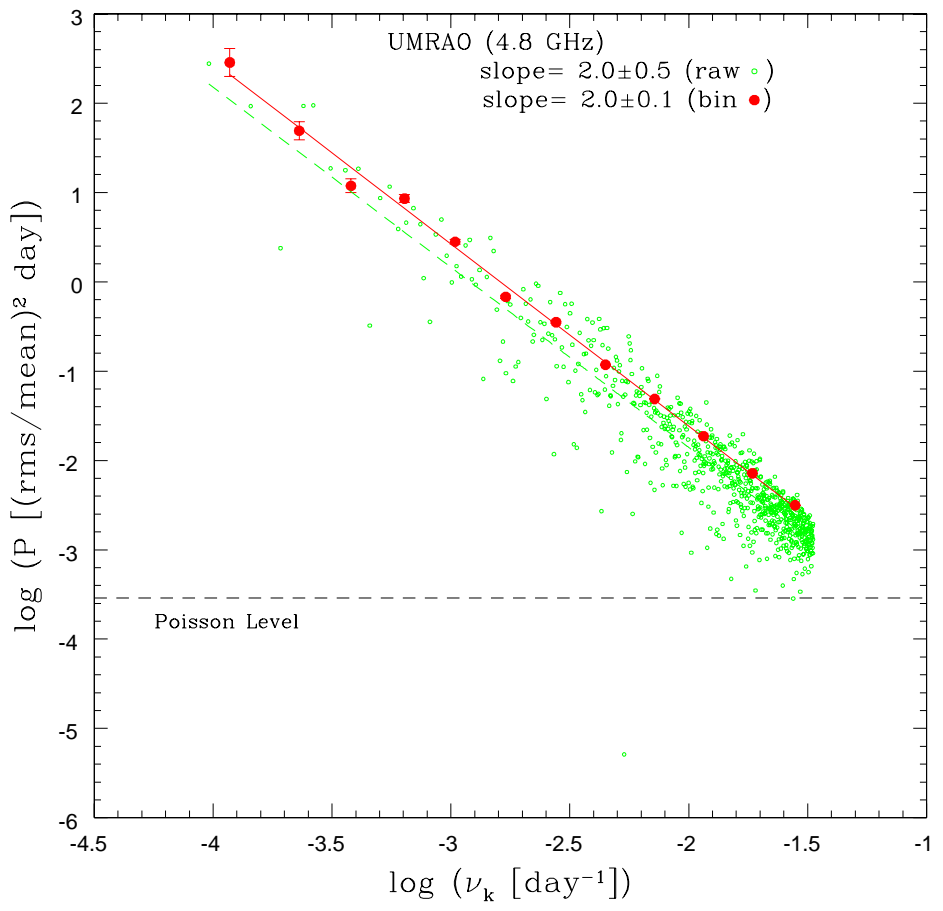

(b)

Figure 1. Cont. 


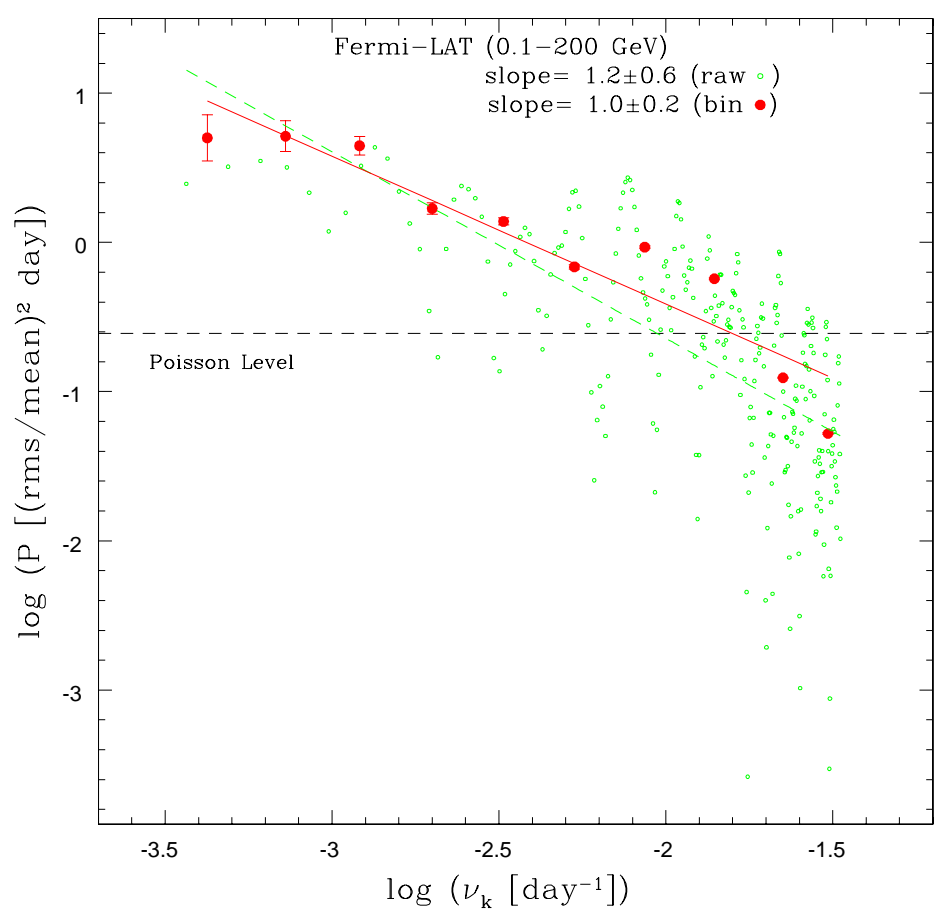

(c)

Figure 1. (a,b) Power spectral densities (PSDs) corresponding to GHz band radio lightcurves of PKS $0735+178$ obtained from the Owens Valley Radio Observatory (OVRO) (total time span of $\sim 7$ years and typical 3 days sampling) and the University of Michigan Radio Astronomy Observatory (UMRAO) (total time span of $\sim 33$ years and typical 15 days sampling) monitoring programmes; (c) The PSD corresponding to the $\gamma$-ray (Fermi-LAT) lightcurve of the source shown in Figure 3a, covering a time span of $\sim 7$ years with 15-day bins. In all panels, empty and filled symbols denote the raw and binned periodogram estimates, respectively, while the dashed horizontal line shows the Poisson noise level due to measurement uncertainty achieved in these observations.

(2) The PSD slopes emerging from our analysis of the long-term Fermi-LAT, optical, and radio light curves of the source on timescales ranging from many years to weeks indicates that the statistical character of $\gamma$-ray flux changes ( $\beta \sim 1$; flickering/pink noise) is different than that of radio and optical flux changes ( $\beta \sim 2$; strict red noise). The PSD normalization is also different for $\gamma$-rays when compared to the PSD at radio/optical frequencies, indicating more variability power in $\gamma$-ray fluctuations on similar timescales (cf. Figures 1 and 2).

As discussed in [11], the red noise can be generated by a single stochastic process with a relaxation timescale longer than variability timescales probed by the source lightcurve, while the pink noise can be generated by a superposition of two stochastic processes, within the range of variability timescales in between the two corresponding relaxation timescales. The PSDs of PKS 0735+178 constructed here could therefore be explained by hypothesizing (in the framework of the model by [11]) that the $\gamma$-ray variability of the source is shaped by a linear superposition of two stochastic processes: (i) the same process that drives the optical and radio variability, characterized by a long relaxation time on the order of decades; and (ii) an additional process characterized by a much shorter relaxation timescale of the orders of (at most) days. We speculate further that the driver behind the former process (i) is related to the complex topology of the coiled and reversing jet magnetic field, supplied by the accretion disk and modified further along the outflow by turbulent cascading (from large to small scales), and also by magnetic reconnection (leading to the formation of merging magnetic islands/tubes). The latter process (ii), on the other hand, may be related to the inhomogeneous distribution of the target photons for the IC scattering within the outflow (resulting from both inhomogeneous distribution of the energy 
dissipation sites, but also relative differences in bulk velocities of various sub-volumes of the jet). If correct, this interpretation indicates that the radio-to- $\gamma$-ray emission of PKS $0735+178$ cannot be explained by a simple "one-zone" synchrotron self-Compton (SSC) model typically applied to BL Lacs.

(3) The optical PSDs on intra-night timescales (for the nights during which the INV has been detected) shows a range of slopes within the range $1<\beta<3$. In all cases, however, the normalization (when accounting for the "red-noise leakage" effect) turns out consistent with a simple extrapolation of the pure red-noise type optical PSD from lower variability frequencies (Figure 2). This indicates that the main driver behind the optical variability of the jet is the same on years, months, days, and hours timescales. This argues against the scenario where different drivers behind the long-term and intra-night flux changes are considered, such as internal shocks due to the jet bulk velocity fluctuation (shaping long-term flux changes) versus small-scale magnetic reconnection events taking place at the jet base (causing intra-night flux changes). However, we note that relaxation timescales corresponding to magnetic field stresses in the reconnection regions could be driven by large-scale modulations in the jet.

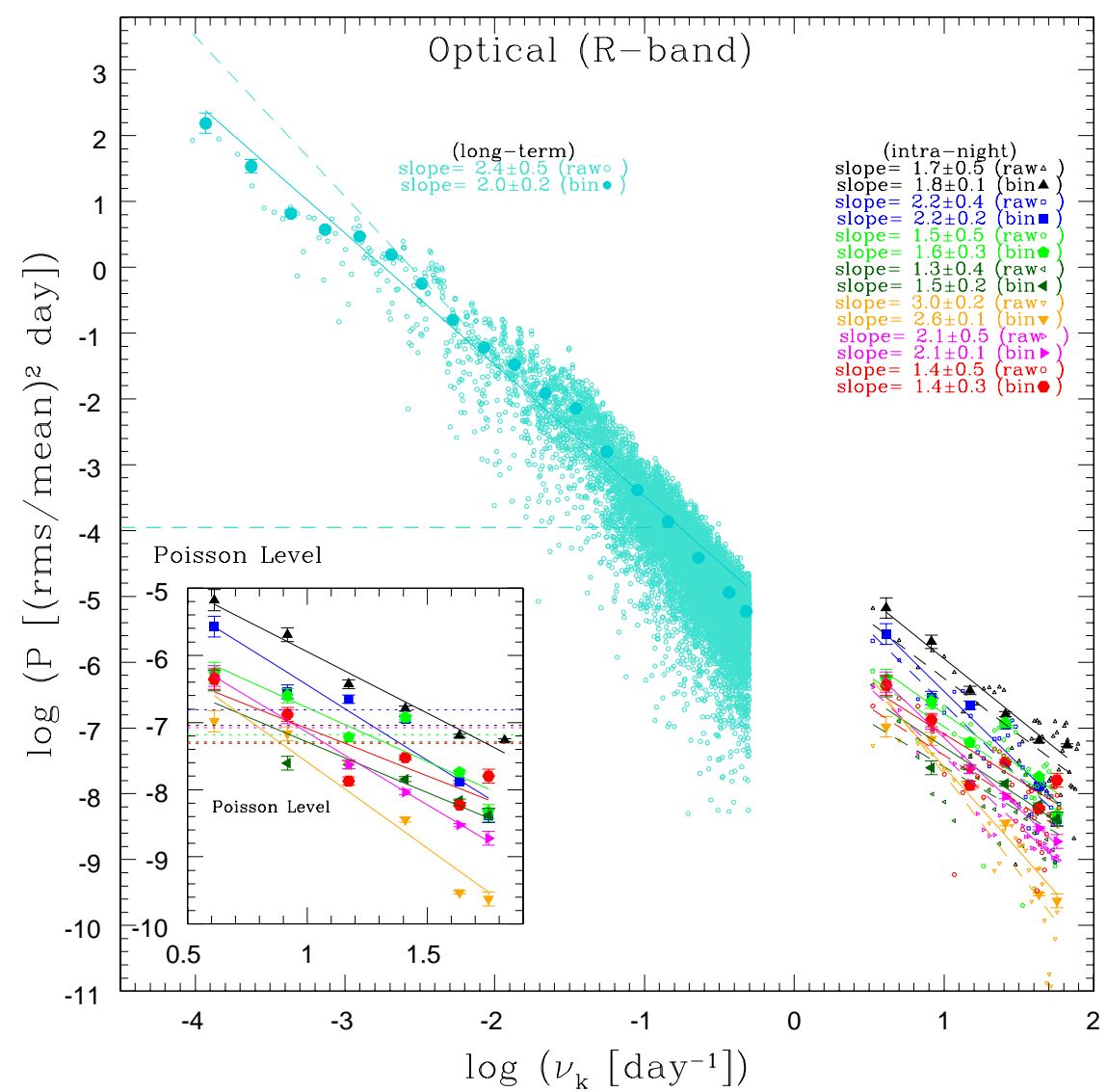

Figure 2. Composite (LTV+INV optical R-band PSD of PKS 0735+178. Long-term optical data were obtained with typical nightly sampling, while the densely-sampled intra-night data were obtained with 10-20 min binning, depending on the source brightness and the sky transparency. Empty and filled symbols show the raw and binned periodogram estimates, respectively, while the dashed horizontal line shows the Poisson noise level due to measurement uncertainty achieved in these observations. Note that R-band PSD includes PSDs drawn using intra-night light curves when confirmed INV was seen (not shown here, see Table 1 of Goyal et al., 2016, submitted). Turquoise shows long-term flux measurements (Figure 3b), while red (10-12-04), magenta (23-12-04), orange (05-01-05), dark green (09-01-05), green (15-12-07), blue (07-01-13), and black (23-12-13) show INV PSDs (epoch of monitoring is given in the parentheses: dd-mm-yy). Inset plot shows the zoomed-in bin INV PSDs for better visibility. 


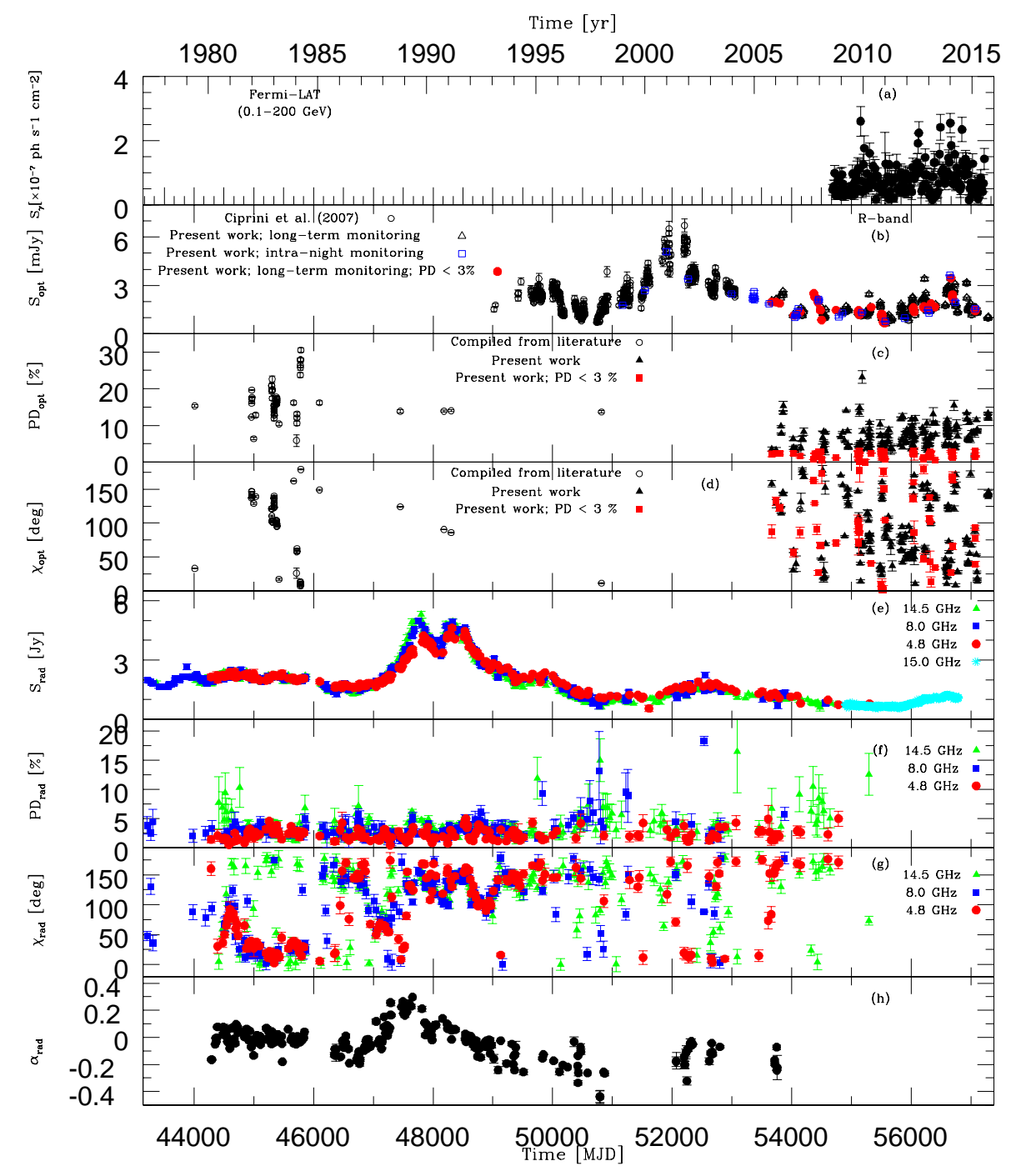

Figure 3. The long-term variability (LTV) lightcurve of BL Lac object PKS 0735+178. Panels a-d show the Fermi-LAT, R-band photometric flux, $P D_{o p t}$, and $\chi_{\text {opt }}$ light curves, respectively; Panels $\mathbf{d}-\mathbf{f}$ show the radio flux at different frequencies, $P D_{\text {rad }}$, and $\chi_{\text {rad }}$, respectively; Panel $\mathbf{h}$ shows the run of radio spectral index (defined as $S_{v} \propto v^{\alpha}$ ) calculated by a linear regression analysis of the flux values at the three frequencies (note that measurements at these frequencies were treated as simultaneous if they occurred within 14 days; i.e., $q=0.04$ year).

We note that our interpretation of the different shapes of the PSDs at $\gamma$-rays and lower frequencies is not unique. An alternative interpretation could be, for example, that $\gamma$-rays originate in hadronic emission processes, or that the emission regions dominating the $\gamma$-ray and lower frequency fluxes are distinct. Those alternative interpretations, while possibly consistent with a difference in PSDs, could not, however, account for the fact why the low frequency PSDs are of exactly the red noise type while the $\gamma$-ray PSD is of exactly the pink noise type. A detailed discussion of the data, data analysis, and interpretation will be presented in the forthcoming paper by Goyal et al., (2016; submitted).

Acknowledgments: Arti Goyal, Łukasz Stawarz and Michal Ostrowski ackowledges support of the Polish National Science Centre through the grant DEC- 2012/04/A/ST9/00083. Valeri Larionov acknowledges the support of Russian RFBR grant 15-02-00949 and St. Petersburg University research grant 6.38.335.2015. A kind thanks to Margo and Hugh Aller for providing the data. Data from the Steward Observatory spectropolarimetric monitoring project were used. This program is supported by Fermi Guest Investigator grants NNX08AW56G, NNX09AU10G, NNX12AO93G, and NNX15AU81G. The OVRO 40 M Telescope Fermi Blazar Monitoring Program is supported by NASA under awards NNX08AW31G and NNX11A043G, and by the NSF under 
awards AST-0808050 and AST-1109911. Boston University data acknoledges the support from National Science Foundation under grant numbers AST-980294, AST-0098579, and AST-0406865.

Author Contributions: Arti Goyal coordinated the optical intra-night observations and analysis, Fermi-LAT data analysis; Power Spectral Density (PSD) analysis and wrote the paper; Łukasz Stawarz and Michal Ostrowski were involved in intrepretation of the results while Valeri Larionov coordinated the polarimetric monitoring and analysis.

Conflicts of Interest: The authors declare no conflict of interest.

\section{References}

1. Urry, C.M.; Padovani, P. Unified Schemes for Radio-Loud Active Galactic Nuclei. Publ. Astron. Soc. Pac. 1995, doi:10.1086/133630.

2. Gopal-Krishna; Wiita, P.J. Swinging jets and the variability of active galactic nuclei. Astron. Astrophys. 1992, 259, 109-117.

3. Marscher, A.P. Turbulent, Extreme Multi-zone Model for Simulating Flux and Polarization Variability in Blazars. Astrophys. J. 2014, doi:10.1088/0004-637X/780/1/87.

4. Sironi, L.; Petropoulou, M.; Giannios, D. Relativistic jets shine through shocks or magnetic reconnection? Mon. Not. R. Astron. Soc. 2015, 450, 183-191.

5. Saito, S.; Stawarz, Ł.; Tanaka, Y.T.; Takahashi, T.; Sikora, M.; Moderski, R. Time-dependent Modeling of Gamma-Ray Flares in Blazar PKS1510-089. Astrophys. J. 2015, doi:10.1088/0004-637X/809/2/171.

6. Goyal, A.; Gopal-Krishna.; Anupama, G.C.; Sahu, D.K.; Sagar, R.; Britzen, S.; Karouzos, M.; Aller, M.F.; Aller, H.D. Unusual optical quiescence of the classical BL Lac object PKS 0735+178 on intranight time-scale. Mon. Not. R. Astron. Soc. 2009, 399, 1622-1632.

7. Ciprini, S.; Takalo, L.O.; Tosti, G.; Raiteri, C.M.; Fiorucci, M.; Villata, M.; Nucciarelli, G.; Lanteri, L.; Nilsson, K.; Ros, J.A. Ten-year optical monitoring of PKS 0735+178: Historical comparison, multiband behavior, and variability timescales. Astron. Astrophys. 2007, 467, 465-483.

8. Goyal, A.; Mhaskey, M.; Gopal-Krishna.; Wiita, P.J.; Stalin, C.S.; Sagar, R. On the Photometric Error Calibration for the Differential Light Curves of Point-like Active Galactic Nuclei. J. Astrophys. Astron. 2013, 34, 273-296.

9. Isobe, N.; Sato, R.; Ueda, Y.; Hayashida, M.; Shidatsu, M.; Kawamuro, T.; Ueno, S.; Sugizaki, M.; Sugimoto, J.; Mihara, T.; et al. MAXI Investigation into the Long-term X-Ray Variability from the Very-high-energy $\gamma$-Ray Blazar Mrk 421. Astrophys. J. 2015, doi:10.1088/0004-637X/798/1/27.

10. Gabuzda, D.C.; Murray, É.; Cronin, P. Helical magnetic fields associated with the relativistic jets of four BL Lac objects. Mon. Not. R. Astron. Soc. 2004, 351, L89-L93.

11. Kelly, B.C.; Sobolewska, M.; Siemiginowska, A. A Stochastic Model for the Luminosity Fluctuations of Accreting Black Holes. Astrophys. J. 2011, doi:10.1088/0004-637X/730/1/52.

(C) 2016 by the authors; licensee MDPI, Basel, Switzerland. This article is an open access article distributed under the terms and conditions of the Creative Commons Attribution (CC-BY) license (http://creativecommons.org/licenses/by/4.0/). 